\title{
Research on Reliability Evaluation Method of Core Processor Software System Based on Component
}

\author{
Zhongwei Chen ${ }^{1, a}$, Songyang Du ${ }^{1, b}$, Xiaoxia Li ${ }^{2, c}$, Qian Guo ${ }^{1, d}$ \\ ${ }^{1}$ Beijing Special Vehicles Institute, Beijing, 100072, China \\ ${ }^{2}$ Beijing Pingguoyuan Middle School, Beijing, 100043, China \\ abigczw@163.com, b ao_0918@163.com, czw_chen@21cn.com, dpaper35413@163.com
}

Keywords: Based on component; Core processor; Software system reliability evaluation.

\begin{abstract}
The integrated electronic information system based on the core processor uses a large number of components which have been finalized or commercially developed, and the system structure is so complicated that common reliability evaluation methods cannot be directly applied to the system. To solve the problem of reliability evaluation of this kind of software system, this paper proposes a reliability evaluation method of core processor software system based on component. Firstly, the core processor software system is analyzed by using the method of system structure to determine the composition form of each component in the system. Then according to the condition of components, reliability evaluation model set will be confirmed and optimized. Finally, the reliability evaluation model of the whole system will be structured and verified. The results showed that the proposed method can achieve a good assessment of the reliability of the software system.
\end{abstract}

\section{Introduction}

The core processor system integrates the voice, data, image and other information with the vehicle control, command and control applications. The existing car terminal, driver terminal, vehicle communication system, network switching equipment, image processing equipment are integrated and functional divided by the core processor system. And then the function of the integrated electronic system of armored vehicle applications can be completed. The core processor software system is deployed in the corresponding device / function module of the core processor system, and it consists of the embedded real-time operating system, the core processor supporting software and a variety of application software. The integrated electronic information system based on the core processor uses a large number of components which have been finalized or commercially developed, so it is important to carry out the reliability evaluation of component based software system.

Due to the complex sources of the application software, we cannot use a single reliability evaluation model to assess the reliability of all components, and we cannot assess the reliability of the system only by the results of the single reliability evaluation model. The present existing selection methods of the comprehensive model are for the whole system, and they regard the whole system as a black box, and discuss the selection of the suitable reliability model for the evaluation system. While when the system is regarded as a white box, and it is discussed that how to choose the suitable evaluation model for each component of the system to ensure system reliability evaluation results more accurate, the related research results are rare.

In order to improve the accuracy of the evaluation results, this paper proposes a reliability evaluation method of core processor software system based on component. Firstly, the method based on the system structure is applied to analyze the core processor software system, and identify the form of components in the system. Then the model selection strategy of component reliability evaluation is determined according to the condition of component. Finally, the reliability of the whole system will be evaluated. 


\section{Core processor software system structure analysis}

The core processor software system is composed of embedded real-time operating system (RTOS), core processor support software (SS) and a variety of application software (US). the overall structure is shown in Figure 1 as the federation structure.

Each core processor (CP) in the system is a federation, and the various federations are combined with distributed structure.

A centralized structure is used inside every single core processor. The core processor supporting software is regarded as the main control software and it is the intermediary of other components while other components are common components. Any two common components must be communicated by the core processor supporting software. Unlike the traditional component, each component in the system is an autonomous component, which has the autonomy, social ability, reactivity, pro-activeness.

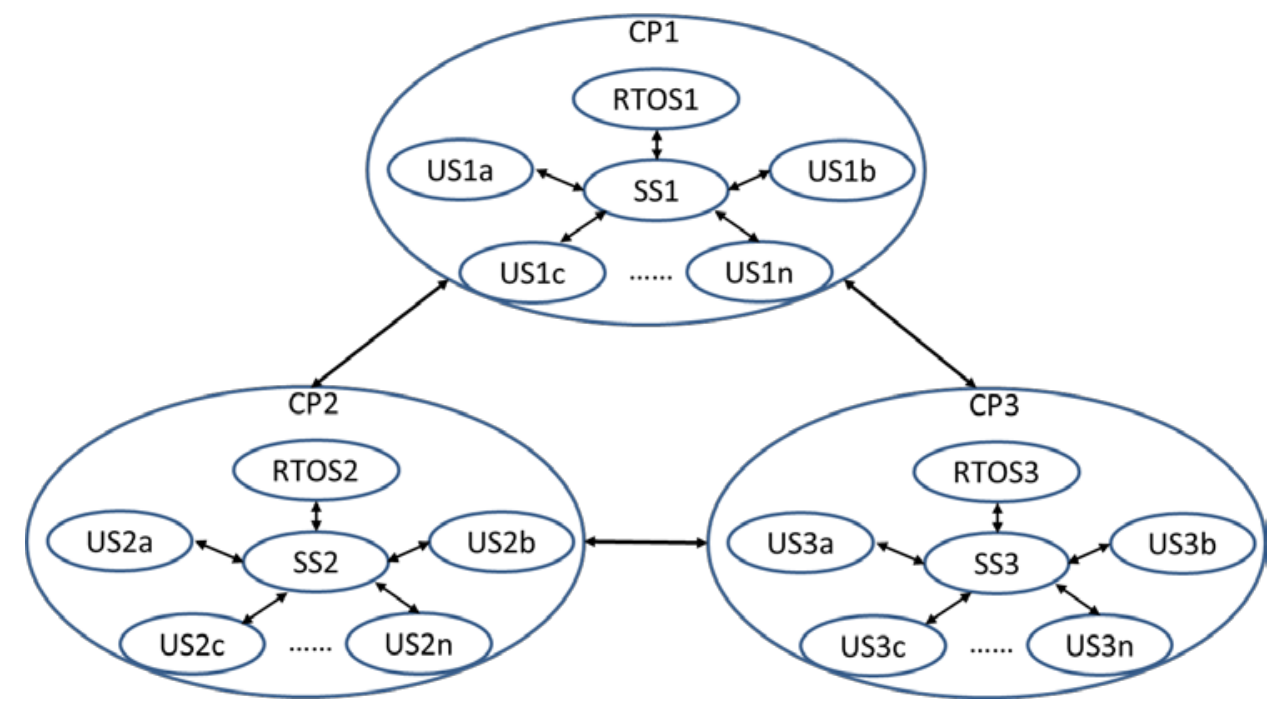

Figure.1. Core processor software system structure

\section{Core processor software system reliability overall evaluation strategy and process}

The core processor software system reliability evaluation can be performed using the process shown in Figure 2.

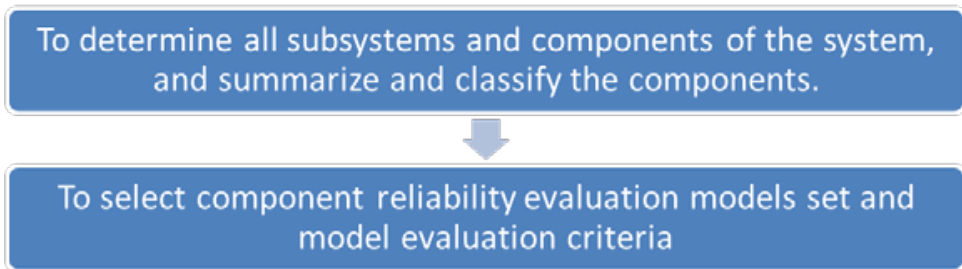

To select the reliability evaluation model of each component, and obtain the evaluation results of each component.

To determine the reliability evaluation model of the system

\section{To calculate the reliability evaluation result of the system.}

Figure.2. The reliability evaluation process of core processor software system

First, we determine all the subsystem of system construction including CP1, CP2, CP3,..., CPn, and determine all components of the subsystems. After the analysis of the demands, the function of 
the system and the component can be determined, and the components to be used can be identified, and then the components can be classified.

Secondly, according to the classification of components, a set of alternative models and the evaluation criteria of each model can be determined.

Again, according to the evaluation criteria, the evaluation value of each component corresponding to different model can be calculated. The evaluation values of the model are compared to find the best fitting model as the reliability evaluation model of the component. Using the selected evaluation model to calculate the reliability of the components, the reliability evaluation results of all the components are obtained.

Then, the system / subsystem reliability evaluation model is structured based on the relationship between the components of the system.

Finally, the reliability of the system is calculated and evaluated according to the evaluation results of the components and their reliability weight factors.

\section{Component reliability evaluation strategy}

Because of the different evaluation results of different data sets by different evaluation model, we can choose the most suitable relative model with different data sets by certain criteria. When the component reliability model is chosen, we must determine the selection criteria first. According to the component reliability data, the evaluation value of each model can be calculated and then the most suitable model for each component can be chosen with the calculation results. There are many proven methods about reliability model selection and calculation, so this paper will not repeat them.

The current model of reliability evaluation for all components can not universally suit for every model. With the complexity and practicability of algorithm considered, this paper uses Mean Square Error (MSE) as evaluation criteria to calculate all candidate models.

$$
\operatorname{MSE}=\frac{1}{\mathrm{n}} \sum_{i=1}^{n}\left[m\left(t_{i}\right)-y_{i}\right]^{2}
$$

In the type $1, m\left(t_{i}\right)$ indicates the number of expected faults detected by time $t_{i}$ by using new model; $y_{i}$ indicates the number of real faults detected by time $t_{i}$. The model coincides with the current data better, and the MSE value should be smaller.

\section{Core processor software system evaluation strategy}

When evaluating the reliability of the system, we need to determine the mathematical model of interaction between the components of the system. There are many reliability evaluation model based on the software architecture, and they are basically divided into four categories, namely state model, path model, component operation profile model and other models. But most models only concerned with the reliability of components, reliability of connectors and transfer ration of components, while they do not consider the key importance of each component for the system. So they are not completely suitable to evaluate the core processor software system with main control software, which need to be further optimized.

When the reliability of the whole system is evaluated, we can first use the centralized structure calculation method to obtain every federation reliability, and then regard every federation as a whole. So, the whole system can be transformed into the form as shown in Figure 3 . Then the reliability of the system can then be calculated by the distributed structure system reliability calculation method. 


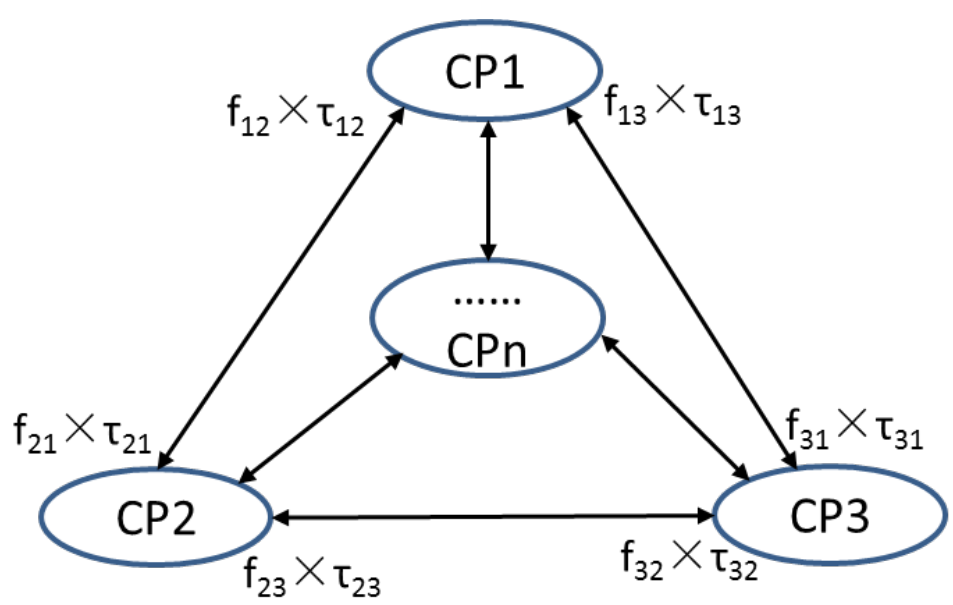

Figure.3. The simplified structure of the core processor software system

In Figure $3, \mathrm{f}_{\mathrm{ij}}$ means transfer ration from federation $\mathrm{CP}_{\mathrm{i}}$ to federation $\mathrm{CP}_{\mathrm{j}}$, and $\tau_{\mathrm{ij}}$ means the communication reliability from $C P_{i}$ to $C P_{j}, i \in[1, n], j \in[1, n]$; $n$ is the number of federation in the system.

For the whole system, every core processor is distributed in the bus network. Thus, when the reliability of the system is evaluated, the reliability of communication should be considered besides the reliability of the component itself. For a single core processor, the supporting software, operating system and application software are all running in the core processor. Thus, a connection structure of objects will be adopted to evaluate, because there is a tight coupling relationship between the components. And then we need not consider the reliability of communication between components.

For the calculation of system reliability of distributed structure, we can directly use CTMC model or DTMC model. CTMC model is suitable for the continuous operation software, and DTMC model is for the software running according to user operation.

\section{Example verification and analysis}

In a certain model vehicle, two types of core processor subsystem were adopted. The supporting software (C1) is as the main control software in the two core processors. Core processor 1 (CP1) is equipped with the operating system (C2), state management software (C3), fault diagnosis software (C4), vehicle display and control software (C5) and others. Core processor 2 (CP2) is equipped with the operating system, state management software, fault diagnosis software, driver display and control software (C6) and others. The reliability of the software system was evaluated synthetically by the proposed method in this article.

The software system consists of 6 components and the alternative models include Yamada Weibull Model (YW), G-O Model (GO), Schneidewind Model. MSE values of each component in each model are shown in Table 1.

Table.1. Evaluation values of each component in the alternative model

\begin{tabular}{|c|c|c|c|}
\hline Component & YW & GO & Schneidewind \\
\hline C1 & 8.05 & 9.41 & 10.57 \\
\hline C2 & 11.62 & 8.57 & 9.24 \\
\hline C3 & 16.59 & 11.29 & 12.32 \\
\hline C4 & 6.13 & 4.71 & 5.28 \\
\hline C5 & 26.04 & 13.82 & 13.39 \\
\hline C6 & 28.77 & 15.98 & 15.04 \\
\hline
\end{tabular}

After optimization, the reliability models are: Yamada Weibull Model (YW) for C1, G-O Model (GO) for C2, C3, and C4, Schneidewind Model for C5 and C6. The reliability calculated by preferred model are: $\mathrm{R}_{\mathrm{C} 1}=0.9909, \mathrm{R}_{\mathrm{C} 2}=0.9876, \mathrm{R}_{\mathrm{C} 3}=0.9279, \mathrm{R}_{\mathrm{C} 4}=0.9878, \mathrm{R}_{\mathrm{C} 5}=0.9653$, $\mathrm{R}_{\mathrm{C} 6}=0.9309$. 
The reliability of CP1 and CP2 can be calculated by using the theoretical model of Markov process, then the system reliability formula is:

$$
\mathrm{R}_{\mathrm{cp}}=\mathrm{R}_{1} \sum_{i=2}^{5} \pi_{\mathrm{i}} \mathrm{R}_{\mathrm{i}}
$$

In type $2, \pi_{\mathrm{i}}$ is probability of each component used in the system.

The calculated reliability according to the above type is: $\mathrm{R}_{\mathrm{cp} 1}=0.9596, \mathrm{R}_{\mathrm{cp} 2}=0.9637$.

Since there are only two core processors in this system, the probability of the two is equal, and it is a series structure. So the reliability of the whole system is obtained as $\mathrm{R}=0.9616$.

\section{Conclusion}

This paper studies the structure and characteristics of the core processor software system, presents the optimized process of a reliability evaluation model. It synthetically applies the various existing software system reliability evaluation methods and establishes the comprehensive evaluation model of the core processor software system reliability. And it proves the effectiveness of the proposed method by the study of specific examples.

The results of this paper can be used to compare, select and design the system architecture based on component, but can also be used to evaluate the reliability of the system when the existing component is used to establish a new system. But because of the simple calculation, component redundancy is not sufficient, and it affects the accuracy of reliability evaluation, which will become the focus in the next research direction.

\section{References}

[1] Minyan Lu. Software Reliability Engineering, 2011.

[2] Nie Peng. Research on key technologies in component-based software heuristic testing and reliability, 2012.

[3] Weimin Shi. Overview on architecture-based software reliability evaluation [J]. Computer Engineering And Design, 2015 36(2) 419-425.

[4] Darong Huang, Xing Han, Hui Jiang. Research on the Integrated Evaluation Model of Software System Reliability [J]. Command Control \& Simulation, 200830 (6) 82-84.

[5] Wei Zhang, Weiqun Zhang. Study of Improved Component-based Software Reliability Model Based on Route [J]. Computer Science, 201138 (2) 148-151. 